\title{
The Effect of Treatment Position on Rectal and Bladder Dose-Volume Histograms for Prostate Radiotherapy Planned with 3-Dimensional Conformal Radiotherapy, Intensity-Modulated Radiotherapy and Volumetric Modulated Arc Therapy
}

\author{
Kotaro Terashima ${ }^{1}$, Katsumasa Nakamura' ${ }^{1}$, Tomonari Sasaki ${ }^{1}$, Saiji Ohga ${ }^{1}$, \\ Tadamasa Yoshitake1, Kazushige Atsumi2, Makoto Shinoto3, Kaori Asai1, \\ Keiji Matsumoto' ${ }^{1}$, Hidenari Hirata ${ }^{1}$, Yoshiyuki Shioyama ${ }^{3}$, Akihiro Nishie ${ }^{1}$, Hiroshi Honda ${ }^{1}$ \\ ${ }^{1}$ Department of Clinical Radiology, Graduate School of Medical Sciences, Kyushu University, Fukuoka, Japan \\ ${ }^{2}$ Department of Radiology, Kyushu University Hospital at Beppu, Beppu, Japan \\ ${ }^{3}$ Kyushu International Heavy Ion Beam Therapy Center, Tosu, Saga, Japan \\ Email: nakam@radiol.med.kyushu-u.ac.jp
}

Received 13 January 2014; revised 12 February 2014; accepted 10 March 2014

Copyright (C) 2014 by authors and Scientific Research Publishing Inc.

This work is licensed under the Creative Commons Attribution International License (CC BY).

http://creativecommons.org/licenses/by/4.0/

c) (7)

Open Access

\begin{abstract}
Purpose: To compare target coverage and organ at risk (OAR) sparing in the supine and prone positions with 3-dimensional conformal radiotherapy (3DCRT), intensity-modulated radiotherapy (IMRT) and volumetric modulated arc therapy (VMAT) in low- and high-risk prostate radiotherapy cases. Materials and Methods: Using magnetic resonance images of five healthy volunteers, six treatment plans (supine 3DCRT, prone 3DCRT, supine IMRT, prone IMRT, supine VMAT and prone VMAT) were generated. Planning target volume 1 (PTV1) was defined as the prostate gland plus the seminal vesicles with adequate margins in a high-risk setting, while PTV2 was defined as prostate only with margins in a low-risk setting. The mean dose for both PTV1 and PTV2 was set at 78 Gy. Plans generated by each of the 3 techniques were compared between the supine and prone positions using dose-volume histograms (DVHs). Results: For PTV1, prone 3DCRT provided a significantly higher D98\% than did supine 3DCRT, and its homogeneity index (HI) was significantly better. IMRT and VMAT values did not differ significantly between the prone and supine positions. For PTV2, no values differed significantly between the supine and prone positions under any treatment plan. With respect to OAR, the rectal Dmean, D2\%, V50, and V60 values of PTV1 were

How to cite this paper: Terashima, K., et al. (2014) The Effect of Treatment Position on Rectal and Bladder Dose-Volume Histograms for Prostate Radiotherapy Planned with 3-Dimensional Conformal Radiotherapy, Intensity-Modulated Radiotherapy and Volumetric Modulated Arc Therapy. International Journal of Medical Physics, Clinical Engineering and Radiation Oncology, 3, 88-97. http://dx.doi.org/10.4236/ijmpcero.2014.32014
\end{abstract}


statistically higher in supine 3DCRT than in prone 3DCRT, while there were no significant differences in rectal values between the supine and prone positions with IMRT or VMAT. The rectal Dmean, V50, V60, V70, and V75 values of prone 3DCRT were significantly higher than those of supine IMRT or supine VMAT. There were no significant differences in any values for the rectum and bladder for PTV2. Conclusion: Although prone 3DCRT was found to be superior to supine 3DCRT in terms of rectal sparing in high-risk prostate cancer, IMRT and VMAT techniques could possibly cover this disadvantage.

Keywords

Prostate Radiotherapy, Dosimetric Comparison, 3DCRT, IMRT, VMAT

\section{Introduction}

External beam radiation therapy (RT) for prostate cancer has been widely used as an important option for radical treatment, and higher dose delivery to the prostate has been known to improve outcome. Although advanced radiation treatment techniques are available, such as 3-dimensional conformal RT (3DCRT), intensity-modulated RT (IMRT), and volumetric modulated arc therapy (VMAT), rectal toxicity is amplified with increased radiation doses.

The selection of treatment position is a critical choice in RT. The choice of position can alter the external contour of the treated area and has the potential to alter the spatial relationship between internal organs. Several authors have demonstrated that the rectal dose is reduced in the prone position [1]-[3]. However, in most previous reports, rectal doses were evaluated in patients treated with 3DCRT, and the effect of patient position on normal tissue doses in IMRT or VMAT is not well known.

The aim of the present study was to compare target coverage and organ at risk (OAR) sparing in the supine and prone positions with 3DCRT, IMRT and VMAT in low- and high-risk cases in order to determine whether differences in the spatial relationship between the critical organs and the target would have an impact on dosimetric improvement in treatment planning.

\section{Materials and Methods}

\subsection{Patient Population}

Five healthy male volunteers were included in the present study. All volunteers gave their informed consent before the investigation began, after being provided with a detailed explanation of the scope and methods to be used. Each volunteer was asked not to empty his bladder for 1 hour before the imaging sessions. No rectal protocol was specified before the imaging sessions.

For treatment planning, 3-tesla magnetic resonance (MR) images were used because we anticipated an increase in the accuracy of delineation of the target or organs. In addition, there was no radiation exposure for normal volunteers.

MRI scans were made of each volunteer in the prone and supine positions on a flat board. No immobilization devices were used. The scans were performed with a 3T Achieva Quasar Dual (Philips Medical Systems, Best, The Netherlands) using T2-weighted fast spin-echo (repetition time/echo time, 4930 - $5620 \mathrm{msec} / 90 \mathrm{msec} ; 90^{\circ}$ flip angle; length, $21 \mathrm{ET}$; 2.6 SENSE factor; no fat suppression; thickness, $2 \mathrm{~mm}$; gap, $0 \mathrm{~mm}$ ). An image data acquisition matrix of $490 \times 490$ or $448 \times 436$ was used with a rectangular field of view of $400 \times 400 \mathrm{~mm}$ or 360 $\times 360 \mathrm{~mm}$. Acquisition time was from 5 minutes 35 seconds to 7 minutes 7 seconds. MR images were transferred to the treatment planning system (ECLIPSE ${ }^{\circledR}$ version 10.0; Varian Medical Systems, Inc., Palo Alto, CA, USA), and all structures were postulated as water-equivalent material. The supine scan was performed initially with the prone scan following immediately.

\subsection{Target and OAR Delineation}

The prostate, seminal vesicles, rectum and bladder were delineated by one of the investigators to minimi-zepo- 
tential interobserver variations. Clinical target volume 1 (CTV1) consisted of the prostate gland plus the seminal vesicles in a high-risk setting, while CTV2 was defined as prostate only in a low-risk setting. Planning target volumes PTV1 and PTV2 included CTV1 and CTV2, respectively, plus an 8-mm safety margin, except at the prostate gland-rectum interface, where a 5-mm margin was used. The bladder and rectum were considered to be solid organs, and the outer circumference was drawn. The bladder was contoured from its base to the dome, and the rectum from $1.0 \mathrm{~cm}$ above the upper end of PTV1 to $1.0 \mathrm{~cm}$ below the lower end of PTV1.

\subsection{Treatment Planning}

Six treatment plans (supine 3DCRT, prone 3DCRT, supine IMRT, prone IMRT, supine VMAT and prone VMAT) were generated for each volunteer. With a conventional fractionation scheme (39 daily fractions of 2 Gy), a dose of 78 Gy was prescribed as the mean dose for PTV1 and PTV2 with $10 \mathrm{~m}$ ega volt X-rays in all plans. The anisotropic analytical algorithm (AAA) version 10.0.28 with a grid size of $2.5 \mathrm{~mm}$ was adopted as the computational algorithm. For 3DCRT plans, a six-field isocentric coplanar technique was planned, as described by Zelefsky et al. [3]. This technique consists of lateral opposed fields and four oblique fields at $35^{\circ}$ from the coronal plane; the plan was to deliver $50 \%$ of the dose laterally and $50 \%$ from the four equally-weighted oblique fields.

For the IMRT plans, a seven-field coplanar with segmental multileaf collimation delivery technique was adopted. Plans were optimized using an inverse planning module. For VMAT via RapidArc (Varian Medical Systems, Palo Alto, CA), one single rotation from $179^{\circ}-181^{\circ}$ in clockwise direction (Varian IEC scale) was used. The collimator was $30^{\circ}$ for all cases. Our plan acceptance criterium for prostate cancer IMRT and VMAT was that more than $95 \%$ of the prescribed dose should be delivered to more than $95 \%$ of the PTV volume (D95\%). The following rectal constraints are used: V75 < 15\%, V70 < 20\%, V65 $<25 \%$, and V60 < 35\% [4]. The $50 \%$ isodose line needed to fall within the rectal contour as much as possible on any individual image slice. For the bladder [5] the constraints were as follows: V75 $<25 \%$, V70 $<35 \%$, and V65 $<50 \%$.

\subsection{Dosimetric Analysis and Plan Comparison}

Plans generated by each of the 3 techniques were compared using dose-volume histograms (DVHs). In compliance with the International Commission on Radiation Units and Measurements (ICRU) 83 report ICRU [6], D98\%, D50\%, and D2\% values were evaluated for PTV1 and PTV2. In accordance with ICRU guidelines [6], the homogeneity index (HI) was calculated as follows:

$$
\mathrm{HI}=(\mathrm{D} 2 \%-\mathrm{D} 98 \%) / \mathrm{D} 50 \%
$$

To characterize the degree of dose conformity, the Dice similarity coefficient (DSC) was used [7]:

$$
\mathrm{DSC}=2 \times(\mathrm{TV} \cap \mathrm{PTV}) /(\mathrm{TV}+\mathrm{PTV})
$$

In Equation (2), TV was the treated volume. The optimal value for DSC is 1. D98\% was used to delineate the TV according to the ICRU guidelines.

For the OAR, several dosimetric indexes were considered: V20, V50, V60, V70, and V75 were the OAR volumes (expressed as a percentage) that received doses of at least 20, 50, 60, 70, and $75 \mathrm{~Gy}$, respectively. The mean dose and the near-maximum absorbed dose (D2\%) were also recorded. The patient-averaged DVHs for PTV1, PTV2, and rectal and bladder values were compared in the supine and prone positions.

For statistical analysis, results were compared using a two-sided paired t-test. The threshold for statistical significance was set at $\mathrm{P}<0.05$. All statistical analyses were carried out using JMP Version 5 (SAS Institute, Cary, NC, USA).

\section{Results}

Table 1 shows the dose characteristics for CTV1, CTV2, PTV1, PTV2, rectum and bladder. No significant differences in volumes were noticed except for the bladder.

For PTV1 (Table 2(a)), prone 3DCRT provided a significantly higher D98\% than did supine 3DCRT, and HI was significantly better in prone 3DCRT. D2\%, D50\%, and DSC values planned by 3DCRT did not differ significantly. Additionally, no values planned by IMRT and VMAT differed significantly between the prone and supine positions. For PTV2 (Table 2(b)), no values differed significantly between supine and prone positions in 
Table 1. Structure volume information for the supine and prone positions.

\begin{tabular}{cccc}
\hline & Supine & Prone & $p$-value \\
\hline CTV1 volume $\left(\mathrm{cm}^{3}\right)$ & $39 \pm 12.0(21.7-50.6)$ & $40.0 \pm 12.8(21.7-53.4)$ & n.s \\
PTV1 volume $\left(\mathrm{cm}^{3}\right)$ & $128.3 \pm 23.9(94.2-148.9)$ & $134.2 \pm 31.1(87.9-164)$ & n.s \\
CTV2 volume $\left(\mathrm{cm}^{3}\right)$ & $27.1 \pm 11.1(13.0-40.2)$ & $25.6 \pm 10.3(15.4-41.7)$ & $79.5 \pm 21.0(56.8-109.9)$ \\
PTV2 volume $\left(\mathrm{cm}^{3}\right)$ & $82.74 \pm 23.6(51.9-106.3)$ & $43.5 \pm 19.5(21.3-68.6)$ & n.s \\
Rectal volume $\left(\mathrm{cm}^{3}\right)$ & $38.7 \pm 20.3(21.4-71.6)$ & $257.9 \pm 111.3(152.5-425.9)$
\end{tabular}

Abbreviations: CTV = clinical target volume; PTV = planning target volume; n.s. = not significant.

any of the three treatment plans.

With respect to OAR, the rectal D2\%, V50, and V60 values of PTV1 were statistically higher with supine 3DCRT than with prone 3DCRT. When supine and prone positions with IMRT or VMAT were compared, there tended to be higher rectal values in supine position, however there was no significant difference in any rectal value (Table 3(a)). For the bladder, only D2\% in prone 3DCRT was higher than supine 3DCRT. No significant differences in other values were observed between the supine and prone positions (Table 4(a)). For PTV2 (Table 3(b), Table 4(b)), there was no significant difference in any value for the rectum and bladder, although there tended to be a higher irradiated volume of the rectum in the supine position, especially in 3DCRT.

Because differences in rectal dose were noted between prone 3DCRT and supine 3DCRT for PTV1, we compared rectal values for prone 3DCRT, supine IMRT and supine VMAT. The rectal Dmean, V50, V60, V70, and V75 values for prone 3DCRT were found to be significantly higher (Table 5). Although the rectal V20 value for 3DCRT was lower than that for IMRT or VMAT, the differences were not significant, and supine IMRT and VMAT were found to be superior to prone 3DCRT in terms of rectal sparing. Compared to supine IMRT, supine VMAT provided significantly better rectum wall sparing for V50 and V60, but not for D2\% and V75, which were not significant.

\section{Discussion}

There has been a debate over the supine versus prone position for some years, and the published literature demonstrates variations in results between the use of these positions for prostate cancer radiotherapy. Several authors have demonstrated that, with 3DCRT, the rectal dose is reduced in the prone position [1] [2] [8]. Therefore, from the viewpoint of dose reduction to the rectum, the prone position may be recommended in patients treated with 3DCRT. We also found that prone 3DCRT was associated with better rectal dose reduction than supine 3DCRT, especially in cases of including seminal vesicles in the CTV. It is considered that seminal vesicle position variability based on position may possibly affect this result. The position of the seminal vesicles in the supine position, which affects the PTV1 area, may be associated with the lower D98\% and higher HI of PTV1 compared to values in the prone position. In contrast, this is not the case with IMRT. Kato et al. [9] compared rectal dose between supine 3DCRT, prone 3DCRT, and supine IMRT, concluding that, although prone 3DCRT resulted in significant improvements in some patients compared with supine 3DCRT, supine IMRT was significantly superior to prone 3DCRT. In the present study, we also found that supine IMRT and VMAT were superior to prone 3DCRT for rectum sparing. Between supine IMRT and supine VMAT, rectal V50 and V60 with supine VMAT were superior to that with supine IMRT which is consistent with the results of previous reports [10]-[12].

There was a significant difference in bladder volume between the supine and prone positions. Because the supine scan was performed initially with the prone scan following in the present study, this result may have been due to increased bladder filling during examination. In the present study, the results for bladder sparing did not show significant differences between the prone and supine positions except in the D2\% value of 3DCRT in PTV1, which might have been affected by bladder volume.

McLaughlin et al. [8] found smaller differences in percent rectal volume at $>80 \%$ and $>90 \%$ of the isocenter dose for prone versus supine positioning for prostate-only treatments compared to prostate and seminal vesicle 
Table 2. (a) Summary of the dosimetric results for PTV1; (b) Summary of the dosimetric results for PTV2.

(a)

\begin{tabular}{|c|c|c|c|c|c|c|}
\hline & & \multicolumn{2}{|c|}{ Supine } & \multicolumn{2}{|c|}{ Prone } & \multirow[b]{2}{*}{$p$-value } \\
\hline & & Ave. & Range (min - max) & Ave. & Range (min - max) & \\
\hline \multirow{5}{*}{ 3DCRT } & D98\% & 74.35 & $74.11-74.71$ & 74.78 & $74.44-75.08$ & 0.049 \\
\hline & D2\% & 79.71 & $79.32-80.47$ & 79.45 & $79.02-79.90$ & n.s \\
\hline & D50\% & 78.30 & 78.14 - 78.39 & 78.26 & $78.16-78.36$ & n.s \\
\hline & $\mathrm{HI}$ & 0.068 & $0.061-0.070$ & 0.060 & $0.053-0.070$ & 0.007 \\
\hline & DSC & 0.728 & $0.701-0.760$ & 0.753 & $0.706-0.808$ & n.s \\
\hline \multirow{5}{*}{ IMRT } & D98\% & 74.46 & $73.86-74.76$ & 74.65 & $74.22-75.19$ & n.s \\
\hline & $\mathrm{D} 2 \%$ & 80.14 & $80.02-80.21$ & 80.29 & $79.86-81.29$ & n.s \\
\hline & D50\% & 78.16 & $78.09-78.22$ & 78.09 & $77.93-78.18$ & n.s \\
\hline & $\mathrm{HI}$ & 0.073 & $0.068-0.081$ & 0.072 & $0.063-0.091$ & n.s \\
\hline & DSC & 0.897 & $0.886-0.911$ & 0.898 & $0.865-0.921$ & n.s \\
\hline \multirow{5}{*}{ VMAT } & D98\% & 75.66 & $74.54-78.15$ & 75.27 & $70.34-77.95$ & n.s \\
\hline & D2\% & 79.73 & $79.49-80.22$ & 80.99 & $79.68-81.79$ & n.s \\
\hline & D50\% & 78.12 & 78.01 - 78.17 & 78.85 & $78.07-79.98$ & n.s \\
\hline & $\mathrm{HI}$ & 0.058 & $0.046-0.068$ & 0.073 & $0.045-0.146$ & n.s \\
\hline & DSC & 0.907 & $0.896-0.925$ & 0.886 & $0.840-0.911$ & n.s \\
\hline
\end{tabular}

(b)

\begin{tabular}{|c|c|c|c|c|c|c|}
\hline & & \multicolumn{2}{|c|}{ Supine } & \multicolumn{2}{|c|}{ Prone } & \multirow[b]{2}{*}{$p$-value } \\
\hline & & Ave. & Range (min - max) & Ave. & Range (min - max) & \\
\hline \multirow{5}{*}{ 3DCRT } & D98\% & 74.36 & $74.07-74.55$ & 74.63 & $74.46-74.84$ & n.s. \\
\hline & $\mathrm{D} 2 \%$ & 79.36 & 79.26 - 79.46 & 79.44 & 79.28 - 79.55 & n.s. \\
\hline & D50\% & 78.34 & 78.32 - 78.37 & 78.34 & $78.24-78.38$ & n.s. \\
\hline & $\mathrm{HI}$ & 0.064 & $0.060-0.069$ & 0.062 & $0.059-0.063$ & n.s. \\
\hline & DSC & 0.728 & $0.615-0.761$ & 0.857 & $0.756-1.219$ & n.s. \\
\hline \multirow{5}{*}{ IMRT } & D98\% & 74.45 & $74.32-74.66$ & 74.56 & $74.27-74.82$ & n.s. \\
\hline & $\mathrm{D} 2 \%$ & 79.50 & $79.43-79.57$ & 79.55 & 79.20 - 79.85 & n.s. \\
\hline & D50\% & 78.32 & 78.25 - 78.39 & 78.30 & $78.24-78.38$ & n.s. \\
\hline & HI & 0.065 & $0.061-0.067$ & 0.064 & $0.056-0.068$ & n.s. \\
\hline & DSC & 0.846 & $0.720-0.917$ & 0.945 & $0.903-1.071$ & n.s. \\
\hline \multirow{5}{*}{ VMAT } & D98\% & 76.23 & $76.01-76.64$ & 75.90 & $75.31-76.18$ & n.s. \\
\hline & D2\% & 79.48 & 79.21 - 79.91 & 79.54 & $79.2-79.89$ & n.s. \\
\hline & D50\% & 78.15 & $78.01-78.67$ & 78.03 & $78.01-78.05$ & n.s. \\
\hline & $\mathrm{HI}$ & 0.046 & $0.039-0.045$ & 0.0466 & $0.041-0.054$ & n.s. \\
\hline & DSC & 0.894 & $0.793-0.922$ & 0.909 & $0.885-0.936$ & n.s. \\
\hline
\end{tabular}

Abbreviations: Ave. = average; HI = homogeneity index; DSC = Dice similarity coefficient; n.s. = not significant; 3DCRT = 3-dimensional conformal radiotherapy; IMRT = intensity-modulated radiotherapy; VMAT = volumetric modulated arc therapy. 
Table 3. (a) Summary of the dosimetric results for rectum planned for PTV1; (b) Summary of the dosimetric results for rectum planned for PTV2.

(a)

\begin{tabular}{|c|c|c|c|c|c|c|}
\hline & & \multicolumn{2}{|c|}{ Supine } & \multicolumn{2}{|c|}{ Prone } & \multirow[b]{2}{*}{$p$-value } \\
\hline & & Ave. & Range (min - max) & Ave. & Range (min - max) & \\
\hline \multirow{7}{*}{ 3DCRT } & D2\% & 79.40 & $79.01-80.11$ & 78.36 & $78.14-78.81$ & 0.002 \\
\hline & Dmean & 59.39 & $55.07-64.27$ & 51.95 & $40.01-56.63$ & n.s. \\
\hline & V20Gy & 90.31 & 86.92 - 91.93 & 84.73 & $76.14-91.04$ & n.s. \\
\hline & V50Gy & 68.94 & $62.24-79.00$ & 55.44 & $34.3-64.62$ & 0.035 \\
\hline & V60Gy & 61.33 & $51.27-74.74$ & 48.64 & $28.19-57.14$ & 0.043 \\
\hline & V70Gy & 56.81 & $40.2-73.32$ & 40.16 & $21.22-48.03$ & n.s. \\
\hline & V75Gy & 39.90 & $31.05-59.96$ & 29.54 & $14.16-36.55$ & n.s. \\
\hline \multirow{7}{*}{ IMRT } & D2\% & 77.95 & $77.25-78.42$ & 77.42 & $75.42-78.70$ & n.s. \\
\hline & Dmean & 44.16 & $44.26-46.88$ & 40.75 & $35.57-43.13$ & n.s. \\
\hline & V20Gy & 89.88 & 88.77 - 91.09 & 86.57 & $82.38-92.33$ & n.s. \\
\hline & V50Gy & 37.58 & $31.41-44.15$ & 30.19 & $17.79-34.65$ & n.s. \\
\hline & V60Gy & 27.21 & $21.18-32.39$ & 22.61 & $12.33-27.59$ & n.s. \\
\hline & V70Gy & 22.43 & $11.28-46.32$ & 14.90 & $7.06-19.28$ & n.s. \\
\hline & V75Gy & 9.29 & $5.54-12.71$ & 9.55 & $3.55-12.11$ & n.s. \\
\hline \multirow{7}{*}{ VMAT } & D2\% & 78.53 & $77.62-79.45$ & 79.06 & $77.27-80.70$ & n.s. \\
\hline & Dmean & 42.45 & $37.91-46.41$ & 40.92 & $32.14-45.63$ & n.s. \\
\hline & V20Gy & 88.06 & $84.48-91.78$ & 86.21 & 81.14 - 91.52 & n.s. \\
\hline & V50Gy & 34.06 & $25.96-40.97$ & 31.24 & $14.14-46.11$ & n.s. \\
\hline & V60Gy & 25.19 & $18.42-30.46$ & 22.94 & 10.35 - 28.67 & n.s. \\
\hline & V70Gy & 16.18 & $11.16-21.56$ & 14.35 & $6.58-20.2$ & n.s. \\
\hline & V75Gy & 10.23 & $6.59-15.36$ & 9.95 & $4.12-14.94$ & n.s. \\
\hline
\end{tabular}

(b)

\begin{tabular}{|c|c|c|c|c|c|c|}
\hline & & \multicolumn{2}{|c|}{ Supine } & \multicolumn{2}{|c|}{ Prone } & \multirow[b]{2}{*}{$p$-value } \\
\hline & & Ave. & Range (min - max) & Ave. & Range (min - max) & \\
\hline \multirow{7}{*}{ 3DCRT } & $\mathrm{D} 2 \%$ & 77.80 & 77.16 - 78.33 & 77.32 & $76.43-77.77$ & n.s. \\
\hline & Dmean & 31.90 & $24.49-42.74$ & 26.02 & $17.24-28.63$ & n.s. \\
\hline & V20Gy & 54.87 & $44.97-71.92$ & 43.49 & $31.05-50.86$ & n.s. \\
\hline & V50Gy & 29.75 & $18.33-42.54$ & 24.27 & $12.73-30.63$ & n.s. \\
\hline & V60Gy & 24.30 & $14.25-35.31$ & 20.27 & $9.94-26.83$ & n.s. \\
\hline & V70Gy & 17.71 & $9.55-27.25$ & 15.15 & $6.7-21.22$ & n.s. \\
\hline & V75Gy & 11.24 & $5.41-19.36$ & 9.30 & $3.52-14.0$ & n.s. \\
\hline
\end{tabular}




\begin{tabular}{|c|c|c|c|c|c|c|}
\hline \multicolumn{7}{|l|}{ Continued } \\
\hline & D2\% & 77.05 & $75.37-78.42$ & 77.54 & 75.96 - 78.54 & n.s. \\
\hline & Dmean & 31.96 & $25.97-39.78$ & 29.24 & $23.01-34.22$ & n.s. \\
\hline & V20Gy & 64.80 & $52.37-78.79$ & 59.61 & $48.46-73.05$ & n.s. \\
\hline \multirow[t]{7}{*}{ IMRT } & V50Gy & 23.11 & $15.91-31.33$ & 19.76 & $12.79-22.75$ & n.s. \\
\hline & V60Gy & 15.67 & $10.06-22.06$ & 13.89 & $8.34-18.96$ & n.s. \\
\hline & V70Gy & 9.10 & $5.18-14.07$ & 8.68 & $4.58-12.88$ & n.s. \\
\hline & V75Gy & 5.19 & $2.32-9.29$ & 5.42 & $2.46-8.82$ & n.s. \\
\hline & D2\% & 77.77 & $76.17-79.14$ & 78.33 & $76.35-79.27$ & n.s. \\
\hline & Dmean & 30.22 & $24.49-38.30$ & 27.32 & $22.23-32.14$ & n.s. \\
\hline & V20Gy & 63.34 & $53.04-78.10$ & 56.44 & $44.82-72.30$ & n.s. \\
\hline \multirow[t]{4}{*}{ VMAT } & V50Gy & 19.71 & $13.19-28.70$ & 17.34 & $10.94-22.93$ & n.s. \\
\hline & V60Gy & 13.61 & $8.65-20.05$ & 12.59 & $7.43-17.31$ & n.s. \\
\hline & V70Gy & 8.50 & $4.87-13.65$ & 8.26 & $4.39-12.18$ & n.s. \\
\hline & V75Gy & 5.48 & $2.73-9.87$ & 5.82 & $2.60-9.00$ & n.s. \\
\hline
\end{tabular}

Abbreviations: Ave. = average; 3DCRT = 3-dimensional conformal radiotherapy; IMRT = intensity-modulated radiotherapy; VMAT = volumetric modulated arc therapy; n.s. = not significant.

treatment. In the present study, although the prone position was found to be superior to the supine position with respect to rectal PTV1 values, there was no significant difference between the supine and prone positions in prostate-only treatment (PTV2), suggesting that the differences in rectal doses between the supine and prone positions were influenced primarily by movement of the seminal vesicles.

In the present study, we used AAA to calculate the dose. Because the same algorithm was used for all dose calculations, the limitation of AAA might not influence the dosimetric comparison.However, a more accurate dose calculation algorithm, such as Acuros XB (available in the Eclipse TPS), had been reported [13] [14], which could further improve the dose calculation accuracy of the dosimetric results.

Sze HC et al. [15] reported that double-arc could improvetarget volume coverage while maintaining equivalent normal tissuesparing when compared with IMRT, and single-arc achieved the greatest treatment efficiency but with the highestrectal dose. Rana S et al. [16] reported the partial-single arc techniquein RapidArc is better in sparing the rectum and bladder without compromising plan conformity or target homogeneity when compared to the full-single arc technique. In this present study, we used seven-field IMRT plans and single-arc VMAT plans, and compared between the prone and supine positions. The selection of techniques such as the number of fields in IMRT, beam angles, and the rotation angle in VMAT might possibly influence the dosimetric results.

In addition to improvements in dose distribution, treatment results may be affected by other factors such as daily setup reproducibility, respiratory motion of the prostate, or rectal filling status. Weber et al. [1] demonstrated that daily setup reproducibility may be less accurate for the prone position, primarily due to systematic setup variations. We previously reported that internal organ motion is less frequent in the supine position than in the prone position using cine-MRI [17]. Treatment results may depend on institution-specific protocols for patient immobilization. Image-guided techniques, which have become widely available in recent years, may reduce interfraction motion.

\section{Conclusion}

The present study demonstrated that, although prone 3DCRT is superior to supine 3DCRT in terms of rectal sparing for high-risk prostate cancer, IMRT and VMAT techniques could possibly cover this disadvantage. With IMRT or VMAT, there was no significant difference between the supine and prone positions. 
Table 4. (a) Summary of the dosimetric results for bladder planned for PTV1; (b) Summary of the dosimetric results for bladder planned for PTV2.

(a)

\begin{tabular}{|c|c|c|c|c|c|c|}
\hline & & \multicolumn{2}{|c|}{ Supine } & \multicolumn{2}{|c|}{ Prone } & \multirow[b]{2}{*}{$p$-value } \\
\hline & & Average & Range (min - max) & Average & Range (min - max) & \\
\hline \multirow{7}{*}{ 3DCRT } & $\mathrm{D} 2 \%$ & 77.85 & $77.54-78.12$ & 78.12 & $77.66-78.42$ & 0.003 \\
\hline & Dmean & 28.3 & $17.71-36.27$ & 27.24 & $15.44-33.15$ & n.s. \\
\hline & V20Gy & 48.75 & $30.08-59.19$ & 46.06 & $24.42-63.30$ & n.s. \\
\hline & V50Gy & 24.06 & $14.33-36.68$ & 25.23 & $12.89-31.71$ & n.s. \\
\hline & V60Gy & 21.90 & $11.5-31.61$ & 21.63 & $10.49-27.74$ & n.s. \\
\hline & V70Gy & 16.51 & $8.17-24.57$ & 16.96 & $7.58-22.36$ & n.s. \\
\hline & V75Gy & 10.53 & $5.27-15.76$ & 12.16 & $5.01-16.54$ & n.s. \\
\hline \multirow{7}{*}{ IMRT } & $\mathrm{D} 2 \%$ & 78.42 & $77.38-78.98$ & 78.36 & $77.58-78.89$ & n.s. \\
\hline & Dmean & 36.67 & $23.24-44.85$ & 31.95 & $17.86-46.88$ & n.s. \\
\hline & V20Gy & 71.32 & $47.48-92.26$ & 59.15 & $32.72-96.13$ & n.s. \\
\hline & V50Gy & 31.15 & $14.71-41.17$ & 27.95 & $13.02-40.5$ & n.s. \\
\hline & V60Gy & 21.65 & $9.76-31.53$ & 19.80 & $9.08-26.25$ & n.s. \\
\hline & V70Gy & 14.66 & $6.13-23.07$ & 13.54 & $6.07-19.09$ & n.s. \\
\hline & V75Gy & 10.40 & $4.0-17.4$ & 9.57 & $4.29-14.34$ & n.s. \\
\hline \multirow{7}{*}{ VMAT } & $\mathrm{D} 2 \%$ & 78.51 & $77.54-79.13$ & 79.18 & $78.23-80.34$ & n.s. \\
\hline & Dmean & 36.68 & $24.1-44.46$ & 32.80 & $17.94-46.64$ & n.s. \\
\hline & V20Gy & 70.91 & $49.48-93.24$ & 58.49 & $32.86-93.79$ & n.s. \\
\hline & V50Gy & 29.09 & $13.58-39.66$ & 28.11 & $11.59-38.73$ & n.s. \\
\hline & V60Gy & 21.34 & $8.94-32.83$ & 20.98 & $7.93-27.45$ & n.s. \\
\hline & V70Gy & 15.07 & $5.3-25.62$ & 14.96 & $5.14-20.94$ & n.s. \\
\hline & V75Gy & 11.14 & $3.61-19.73$ & 11.41 & $3.58-16.77$ & n.s. \\
\hline
\end{tabular}

(b)

\begin{tabular}{|c|c|c|c|c|c|c|}
\hline & & \multicolumn{2}{|c|}{ Supine } & \multicolumn{2}{|c|}{ Prone } & \multirow[b]{2}{*}{$p$-value } \\
\hline & & Average & Range (min - max) & Average & Range (min - max) & \\
\hline \multirow{7}{*}{ 3DCRT } & D2\% & 76.53 & $72.92-77.72$ & 76.98 & $74.73-77.97$ & n.s. \\
\hline & Dmean & 18.89 & $7.96-27.53$ & 17.07 & $8.58-22.07$ & n.s. \\
\hline & V20Gy & 28.79 & $11.09-40.97$ & 25.84 & $12.19-33.55$ & n.s. \\
\hline & V50Gy & 17.29 & $6.01-27.76$ & 15.46 & $6.72-21.88$ & n.s. \\
\hline & V60Gy & 14.18 & $4.57-23.64$ & 12.57 & $5.18-18.39$ & n.s. \\
\hline & V70Gy & 9.99 & $2.76-17.60$ & 8.87 & $3.42-13.5$ & n.s. \\
\hline & V75Gy & 5.95 & $1.27-11.03$ & 5.40 & $1.92-8.52$ & n.s. \\
\hline
\end{tabular}




\begin{tabular}{ccccccc} 
Continued & & & & & \\
\hline & D2\% & 76.44 & $68.59-78.74$ & 76.74 & $71.19-78.59$ & n.s. \\
& Dmean & 18.39 & $7.33-25.35$ & 15.23 & $7.64-21.14$ & n.s. \\
& V20Gy & 31.52 & $11.54-40.63$ & 25.45 & $12.18-39.04$ & n.s. \\
IMRT & V50Gy & 14.03 & $4.45-22.42$ & 11.30 & $4.77-15.36$ & n.s. \\
& V60Gy & 10.27 & $3.04-17.61$ & 8.30 & $3.38-12.06$ & n.s. \\
& V70Gy & 6.79 & $1.83-12.39$ & 5.44 & $2.16-8.31$ & n.s. \\
& V75Gy & 4.61 & $1.09-8.76$ & 3.69 & $1.43-5.79$ & n.s. \\
& D2\% & 77.14 & $71.59-78.88$ & 77.70 & $75.79-78.38$ & n.s. \\
& Dmean & 20.45 & $8.35-27.92$ & 17.22 & $8.89-23.95$ & n.s. \\
& V20Gy & 33.56 & $12.51-42.53$ & 28.07 & $13.68-42.97$ & n.s. \\
& V50Gy & 16.12 & $5.01-25.32$ & 13.13 & $5.85-18.47$ & n.s. \\
& V60Gy & 12.31 & $3.55-20.83$ & 10.06 & $4.41-14.89$ & n.s. \\
& V70Gy & 8.76 & $2.23-16.08$ & 7.18 & $3.04-11.23$ & n.s.
\end{tabular}

Abbreviations: Ave. $=$ average; $3 \mathrm{DCRT}=3$-dimensional conformal radiotherapy; $\mathrm{IMRT}=$ intensity-modulated radiotherapy; VMAT = volumetric modulated arc therapy; n.s. = not significant.

Table 5. Comparisons of rectal dosimetric values for PTV1 between prone 3DCRT, supine IMRT, and supine VMAT.

\begin{tabular}{|c|c|c|c|c|c|c|}
\hline \multirow[t]{2}{*}{ Rectum } & Prone 3DCRT & $\begin{array}{l}\text { Supine } \\
\text { IMRT }\end{array}$ & $\begin{array}{l}\text { Supine } \\
\text { VMAT }\end{array}$ & $\begin{array}{l}\text { Prone 3DCRT vs. } \\
\text { Supine IMRT }\end{array}$ & $\begin{array}{l}\text { Prone 3DCRT vs. } \\
\text { Supine VMAT }\end{array}$ & $\begin{array}{l}\text { Supine IMRT vs. } \\
\text { Supine VMAT }\end{array}$ \\
\hline & Ave. & Ave. & Ave. & $p$-value & $p$-value & $p$-value \\
\hline $\mathrm{D} 2 \%$ & 78.36 & 77.95 & 78.53 & n.s. & n.s. & n.s. \\
\hline Dmean & 51.95 & 44.16 & 42.45 & 0.031 & 0.013 & n.s. \\
\hline V20Gy & 84.73 & 89.88 & 88.06 & n.s. & n.s. & n.s. \\
\hline V50Gy & 55.44 & 37.58 & 34.06 & 0.009 & 0.003 & 0.006 \\
\hline V60Gy & 48.64 & 27.21 & 25.19 & 0.004 & 0.003 & 0.001 \\
\hline V70Gy & 40.16 & 22.43 & 16.31 & 0.030 & 0.003 & n.s. \\
\hline V75Gy & 29.54 & 9.29 & 10.23 & 0.001 & 0.004 & n.s. \\
\hline
\end{tabular}

Abbreviations: Ave. = average; 3DCRT = 3-dimensional conformal radiotherapy; IMRT = intensity-modulated radiotherapy; VMAT = volumetric modulated arc therapy; n.s. = not significant.

\section{Acknowledgements}

This study was supported in part by a Health Labor Sciences Research Grant (H23-Sanjigan-Ippan-007) from the Japanese Ministry of Health, Labor and Welfare and KAKENHI Grants-in-Aid for Scientific Research (Nos. 207004010001, 23390302 and 23659589).

\section{References}

[1] Weber, D.C., Nouet, P., Rouzaud, M. and Miralbell, R. (2000) Patient Positioning in Prostate Radiotherapy: Is Prone Better than Supine? International Journal of Radiation Oncology, Biology, Physics, 47, 365-371.

http://dx.doi.org/10.1016/S0360-3016(99)00458-7

[2] O’Neill, L., Armstrong, J., Buckney, S., Assiri, M., Cannon, M. and Holmberg, O. (2008) A Phase II Trial for the Op- 
timisation of Treatment Position in the Radiation Therapy of Prostate Cancer. Radiotherapy and Oncology: Journal of the European Society for Therapeutic Radiology and Oncology, 88, 61-66.

http://dx.doi.org/10.1016/j.radonc.2008.03.023

[3] Zelefsky, M.J., Happersett, L., Leibel, S.A., Burman, C.M., Schwartz, L., Dicker, A.P., Kutcher, G.J. and Fuks, Z. (1997) The Effect of Treatment Positioning on Normal Tissue Dose in Patients with Prostate Cancer Treated with Three-Dimensional Conformal Radiotherapy. International Journal of Radiation Oncology, Biology, Physics, 37, 1319. http://dx.doi.org/10.1016/S0360-3016(96)00460-9

[4] Michalski, J.M., Gay, H., Jackson, A., Tucker, S.L. and Deasy, J.O. (2010) Radiation Dose-Volume Effects in Radiation-Induced Rectal Injury. International Journal of Radiation Oncology, Biology, Physics, 76, S123-S129. http://dx.doi.org/10.1016/j.ijrobp.2009.03.078

[5] Viswanathan, A.N., Yorke, E.D., Marks, L.B., Eifel, P.J. and Shipley, W.U. (2010) Radiation Dose-Volume Effects of the Urinary Bladder. International Journal of Radiation Oncology, Biology, Physics, 76, S116-S122. http://dx.doi.org/10.1016/j.ijrobp.2009.02.090

[6] The International Commission on Radiation Units and Measurements. Journal of the ICRU, 10. http://dx.doi.org/10.1093/jicru/ndq001

[7] Pasquier, D., Cavillon, F., Lacornerie, T., Touzeau, C., Tresch, E. and Lartigau, E. (2013) A Dosimetric Comparison of Tomotherapy and Volumetric Modulated Arc Therapy in the Treatment of High-Risk Prostate Cancer with Pelvic Nodal Radiation Therapy. International Journal of Radiation Oncology, Biology, Physics, 85, 549-554. http://dx.doi.org/10.1016/j.ijrobp.2012.03.046

[8] McLaughlin, P.W., Wygoda, A., Sahijdak, W., Sandler, H.M., Marsh, L., Roberson, P. and Ten Haken, R.K. (1999) The Effect of Patient Position and Treatment Technique in Conformal Treatment of Prostate Cancer. International Journal of Radiation Oncology, Biology, Physics, 45, 407-413. http://dx.doi.org/10.1016/S0360-3016(99)00207-2

[9] Kato, T., Obata, Y., Kadoya, N. and Fuwa, N. (2009) A Comparison of Prone Three-Dimensional Conformal Radiotherapy with Supine Intensity-Modulated Radiotherapy for Prostate Cancer: Which Technique Is More Effective for Rectal Sparing? The British Journal of Radiology, 82, 654-661. http://dx.doi.org/10.1259/bjr/96404007

[10] Kopp, R.W., Duff, M., Catalfamo, F., Shah, D., Rajecki, M. and Ahmad, K. (2011) VMAT vs. 7-Field-IMRT: Assessing the Dosimetric Parameters of Prostate Cancer Treatment with a 292-Patient Sample. Medical Dosimetry: Official Journal of the American Association of Medical Dosimetrists, 36, 365-372. http://dx.doi.org/10.1016/j.meddos.2010.09.004

[11] Wolff, D., Stieler, F., Welzel, G., Lorenz, F., Abo-Madyan, Y., Mai, S., Herskind, C., Polednik, M., Steil, V., Wenz, F. and Lohr, F. (2009) Volumetric Modulated Arc Therapy (VMAT) vs. Serial Tomotherapy, Step-and-Shoot IMRT and 3D-Conformal RT for Treatment of Prostate Cancer. Radiotherapy and Oncology: Journal of the European Society for Therapeutic Radiology and Oncology, 93, 226-233. http://dx.doi.org/10.1016/j.radonc.2009.08.011

[12] Zhang, P., Happersett, L., Hunt, M., Jackson, A., Zelefsky, M. and Mageras, G. (2010) Volumetric Modulated Arc Therapy: Planning and Evaluation for Prostate Cancer Cases. International Journal of Radiation Oncology, Biology, Physics, 76, 1456-1462. http://dx.doi.org/10.1016/j.ijrobp.2009.03.033

[13] Vassiliev, O.N., Wareing, T.A., McGhee, J., Failla, G., Salehpour, M.R. and Mourtada, F. (2010) Validation of a New Grid-Based Boltzmann Equation Solver for Dose Calculation in Radiotherapy with Photon Beams. Physics in Medicine and Biology, 55, 581-598. http://dx.doi.org/10.1088/0031-9155/55/3/002

[14] Rana, S., Rogers, K., Lee, T., Reed, D. and Biggs, C. (2013) Dosimetric Impact of Acuros XB Dose Calculation Algorithm in Prostate Cancer Treatment Using Rapid Arc. Journal of Cancer Research and Therapeutics, 9, 430-435. http://dx.doi.org/10.4103/0973-1482.119328

[15] Sze, H.C., Lee, M.C., Hung, W.M., Yau, T.K. and Lee, A.W. (2012) Rapid Arc Radiotherapy Planning for Prostate Cancer: Single-Arc and Double-Arc Techniques vs. Intensity-Modulated Radiotherapy. Medical Dosimetry: Official Journal of the American Association of Medical Dosimetrists, 37, 87-91. http://dx.doi.org/10.1016/j.meddos.2011.01.005

[16] Rana, S. and Cheng, C. (2013) Feasibility of the Partial-Single Arc Technique in Rapid Arc Planning for Prostate Cancer Treatment. Chinese Journal of Cancer, 32, 546-552. http://dx.doi.org/10.5732/cjc.013.10077

[17] Terashima, K., Nakamura, K., Shioyama, Y., Sasaki, T., Ohga, S., Nonoshita, T., Yoshitake, T., Atsumi, K., Asai, K., Hirakawa, M., Anai, S., Yoshikawa, H. and Honda, H. (2013) Can a Belly Board Reduce Respiratory-Induced Prostate Motion in the Prone Position?-Assessed by Cine-Magnetic Resonance Imaging. Technology in Cancer Research \& Treatment, 12, 447-453. http://dx.doi.org/10.7785/tcrt.2012.500334 